\title{
New Interval Methodologies for Reliable Chemical Process Modeling
}

\author{
Chao-Yang Gau* and Mark A. Stadtherr ${ }^{\dagger}$ \\ Department of Chemical Engineering \\ University of Notre Dame \\ 182 Fitzpatrick Hall \\ Notre Dame, IN 46556 USA
}

March 2001

(revised, December 2001)

Keywords: Modeling, Nonlinear Equations, Optimization, Numerical Methods, Interval Analysis

*Current address: LINDO Systems, Inc., 1415 North Dayton Street, Chicago, IL 60622, USA

${ }^{\dagger}$ Author to whom all correspondence should be addressed. Fax: (219) 631-8366; E-mail: markst@nd.edu 


\begin{abstract}
The use of interval methods, in particular interval-Newton/generalized-bisection techniques, provides an approach that is mathematically and computationally guaranteed to reliably solve difficult nonlinear equation solving and global optimization problems, such as those that arise in chemical process modeling. The most significant drawback of the currently used interval methods is the potentially high computational cost that must be paid to obtain the mathematical and computational guarantees of certainty. New methodologies are described here for improving the efficiency of the interval approach. In particular, a new hybrid preconditioning strategy, in which a simple pivoting preconditioner is used in combination with the standard inverse-midpoint method, is presented, as is a new scheme for selection of the real point used in formulating the interval-Newton equation. These techniques can be implemented with relatively little computational overhead, and lead to a large reduction in the number of subintervals that must be tested during the intervalNewton procedure. Tests on a variety of problems arising in chemical process modeling have shown that the new methodologies lead to substantial reductions in computation time requirements, in many cases by multiple orders of magnitude.
\end{abstract}




\section{Introduction}

The need to solve systems of nonlinear equations is of course an important issue in the field of scientific and engineering computation. For example, in chemical process modeling there is a frequent need to deal with nonlinear models of physical phenomena and of the manufacturing processes exploiting these phenomena. Nonlinear equation solving problems may arise directly in solving such models, or indirectly in solving optimization problems based on the models. Because of the nonlinearity of the systems to be solved, a number of reliability issues arise. For example, a system may have multiple solutions. In some cases, finding all the solutions may be necessary; in other cases, there may be only one physically correct solution, and some assurance is needed that that solution is not missed. It may also be that a system has no solution. In this situation, it is useful to know with certainty that this is in fact the case, and that failure to find a solution is not due to some numerical or software issue. Furthermore, even if there is only a single solution, it may be difficult to find using standard Newton or quasi-Newton techniques, since the convergence behavior of these methods can be highly initialization dependent.

A wide variety of techniques have been introduced to try to address such reliability issues. For example, trust-region approaches such as the dogleg method (e.g. Powell, 1970; Chen and Stadtherr, 1981; Lucia and Liu, 1998) can improve convergence behavior. Homotopy-continuation methods (e.g., Wayburn and Seader, 1987; Kuno and Seader, 1988; Sun and Seider, 1995; Jalali-Farahani and Seader, 2000) often provide much improved reliability and also the capability for locating multiple solutions. However, all of these approaches are still initialization dependent, and, except in some special cases, can provide no guarantee that all solutions of the nonlinear system will be found. One approach to providing such guarantees is to reformulate the equation solving problem as an optimization problem (e.g. Maranas and Floudas, 1995; Harding et al., 1997; Harding and Floudas, 2000) and then apply powerful deterministic global optimization techniques such as alpha-BB (Adjiman et al., 1998a,b), which employs a branch-and-bound methodology using convex underestimating functions. This provides a mathematical guarantee that all solutions will be located. However, to use this approach it may be necessary to perform problem reformulations and develop convex underestimators specific to each new application.

Another approach for reliable nonlinear equation solving is the use of interval methods, in particular, interval-Newton/generalized-bisection (IN/GB) techniques. This approach is mathematically and computationally guaranteed to find (or, more precisely, to enclose within very narrow intervals) any and all solutions to a system of nonlinear equations. The computational guarantee is possible because the interval 
methodology deals automatically with rounding error issues, and is emphasized since a purely mathematical guarantee may be lost once the technique offering it is implemented in floating point arithmetic. Good introductions to the use of interval methods have been provided by Neumaier (1990), Hansen (1992), and Kearfott (1996). While the use of interval techniques for nonlinear equation solving in process modeling problems was originally explored some time ago (Shacham and Kehat, 1973), it has been only in recent years that the methodology has been more widely studied (e.g., Schnepper and Stadtherr, 1996; Balaji and Seader, 1995). Among the problems to which it has been successfully applied are phase stability analysis (e.g., Stadtherr et al., 1995; McKinnon et al., 1996; Hua et al., 1996, 1998; Xu et al., 2000; Tessier et al., 2000), computation of azeotropes (Maier et al., 1998, 2000), and parameter estimation in vapor/liquid equilibrium models (Gau and Stadtherr, 2000a). Using the interval approach, these problems can be solved with complete certainty. The methodology is general purpose and can be applied to a wide variety of equation solving and optimization problems in process modeling.

The most significant drawback of the currently used interval methods is the potentially high computational cost (CPU time) that must be paid to obtain the mathematical and computational guarantees of certainty. In general, this has limited the size of problems that can be addressed using this methodology, though problems involving over one hundred variables have been successfully solved (Schnepper and Stadtherr, 1996). We describe here new methodologies for improving the efficiency of the interval-Newton approach. The focus is on the formulation and solution of the interval-Newton equation, a linear interval equation system whose solution is a key step in the IN/GB algorithm. In particular, we consider the preconditioning strategy used when solving the interval-Newton equation, and the selection of the real point used in formulating the interval-Newton equation. The new techniques are tested using a variety of problems, leading to large reductions in computation time requirements, in some cases by factors of two orders of magnitude or more.

\section{Background}

\subsection{Interval-Newton Method}

A real interval $X$ is defined as the set of real numbers lying between (and including) given upper and lower bounds; that is, $X=[\underline{X}, \bar{X}]=\{x \in \Re \mid \underline{X} \leq x \leq \bar{X}\}$. Here an underline is used to indicate the lower bound of an interval and an overline is used to indicate the upper bound. A real interval vector 
$\mathbf{X}=\left(X_{1}, X_{2}, \ldots, X_{n}\right)^{\mathrm{T}}$ has $n$ real interval components and can be interpreted geometrically as an $n$ dimensional rectangle or box. Note that in this context uppercase quantities are intervals, and lowercase quantities or uppercase quantities with underline or overline are real numbers.

Of particular interest here is the interval-Newton technique. Given a nonlinear equation system, $\mathbf{f}(\boldsymbol{x})=\mathbf{0}$, with a finite number of real roots in some specified initial interval, this technique provides the capability to find narrow enclosures of all the roots of the system that are within the given initial interval. If the problem is a global optimization problem, say the unconstrained minimization of $\phi(x)$, then the equation system of interest is $\mathbf{f}(\boldsymbol{x})=\nabla \phi(\boldsymbol{x})=\mathbf{0}$. The global minimum will be a root of this nonlinear equation system, but there may be many other roots as well, representing other local minima and maxima and saddle points. Thus the capability to enclose all the roots of the equation system is needed, and this is provided by the interval-Newton technique. In practice, by using the objective range test discussed below, the intervalNewton procedure can be implemented so that roots of $\mathbf{f}(\boldsymbol{x})=\mathbf{0}$ that cannot be the global minimum need not be found. If the global optimization problem is a constrained problem, then the interval-Newton method can be applied to solve the KKT or Fritz-John conditions.

Beginning with some initial interval $\mathbf{X}^{(0)}$ specified by the user, the solution algorithm is applied to a sequence of subintervals. This initial interval can be chosen to be sufficiently large to enclose all physically feasible behavior. For a subinterval $\mathbf{X}^{(k)}$ in the sequence, the first step in the solution algorithm is the function range test. Here an interval extension $\mathbf{F}\left(\mathbf{X}^{(k)}\right)$ of the function $\mathbf{f}(\boldsymbol{x})$ is calculated. An interval extension provides upper and lower bounds on the range of values that a function may have in a given interval. It is often computed by substituting the given interval into the function and then evaluating the function using interval arithmetic. The interval extension so determined is often wider than the actual range of function values, but it always includes the actual range. If there is any component of the interval extension $\mathbf{F}\left(\mathbf{X}^{(k)}\right)$ that does not contain zero, then we may discard the current subinterval $\mathbf{X}^{(k)}$, since the range of the function does not include zero anywhere in this subinterval, and thus no solution of $\mathbf{f}(\boldsymbol{x})=\mathbf{0}$ can exist in this subinterval. We may then proceed to consider the next subinterval in the sequence. Otherwise, if $\mathbf{0} \in \mathbf{F}\left(\mathbf{X}^{(k)}\right)$, then testing of $\mathbf{X}^{(k)}$ continues.

If the problem is a global minimization problem, then the next step is the objective range test. The interval extension $\Phi\left(\mathbf{X}^{(k)}\right)$, which contains the range of $\phi(\boldsymbol{x})$ over $\mathbf{X}^{(k)}$, is computed. If the lower bound of $\Phi\left(\mathbf{X}^{(k)}\right)$ is greater than a known upper bound on the global minimum of $\phi(\boldsymbol{x})$, then $\mathbf{X}^{(k)}$ cannot contain the global minimum and need not be further tested. Otherwise, testing of $\mathbf{X}^{(k)}$ continues. In cases for which 
it is desired to find all the stationary points (or KKT points) rather than just the global minimum, then this step can be turned off.

The next step is the interval-Newton test. Here the linear interval equation system

$$
F^{\prime}\left(\mathbf{X}^{(k)}\right)\left(\mathbf{N}^{(k)}-\boldsymbol{x}^{(k)}\right)=-\mathbf{f}\left(\boldsymbol{x}^{(k)}\right)
$$

is set up and solved for a new interval $\mathbf{N}^{(k)}$, where $F^{\prime}\left(\mathbf{X}^{(k)}\right)$ is an interval extension of the Jacobian of $\mathbf{f}(\boldsymbol{x})$, and $\boldsymbol{x}^{(k)}$ is a point in the interior of $\mathbf{X}^{(k)}$, usually taken to be the midpoint. It has been shown that any root $\boldsymbol{x}^{*}$ contained in $\mathbf{X}^{(k)}$ is also contained in the image $\mathbf{N}^{(k)}$. This implies that if there is no intersection between $\mathbf{X}^{(k)}$ and $\mathbf{N}^{(k)}$ then no root exists in $\mathbf{X}^{(k)}$, and suggests the iteration scheme $\mathbf{X}^{(k+1)}=\mathbf{X}^{(k)} \cap \mathbf{N}^{(k)}$. In addition to this iteration step, which can be used to tightly enclose a solution, it has been proven that if $\mathbf{N}^{(k)}$ is contained completely within $\mathbf{X}^{(k)}$, then there is a unique root contained in the current subinterval $\mathbf{X}^{(k)}$. Thus, after computation of $\mathbf{N}^{(k)}$ from Eq. (1), there are three possibilities: $1 . \mathbf{X}^{(k)} \cap \mathbf{N}^{(k)}=\emptyset$, meaning there is no root in the current subinterval $\mathbf{X}^{(k)}$ and it can be discarded; 2. $\mathbf{N}^{(k)} \subset \mathbf{X}^{(k)}$, meaning that there is a unique root in the current subinterval $\mathbf{X}^{(k)} ; 3$. Neither of the above. In the last case, if the intersection $\mathbf{X}^{(k)} \cap \mathbf{N}^{(k)}$ is sufficiently smaller than $\mathbf{X}^{(k)}$, one can proceed by reapplying the interval-Newton test to the intersection. Otherwise, the intersection is bisected, and the resulting two subintervals added to the sequence of subintervals to be tested. This approach is referred to as an interval-Newton/generalizedbisection (IN/GB) method. At termination, meaning after all subintervals in the sequence (and thus the entire initial search space $\mathbf{X}^{(0)}$ ) have been tested, the result is either enclosures for all the real roots of $\mathbf{f}(\boldsymbol{x})=\mathbf{0}$, or the knowledge that no such roots exist. If desired, this technique can also be extended, as demonstrated, for example, by Balaji and Seader (1995), to locate complex, not just real, zeros of $\mathbf{f}(\boldsymbol{x})$. For additional mathematical details, the monographs of Neumaier (1990), Hansen (1992), and Kearfott (1996) are very useful.

When machine computations with interval arithmetic operations are done, the endpoints of an interval are computed with a directed outward rounding. That is, the lower endpoint is rounded down to the next machine-representable number and the upper endpoint is rounded up to the next machine-representable number. In this way, through the use of interval, as opposed to floating point arithmetic, any potential rounding error problems are eliminated. Overall, the IN/GB method described above provides a procedure that is both mathematically and computationally guaranteed to find narrow enclosures of all solutions of the nonlinear equation system $\mathbf{f}(\boldsymbol{x})=\mathbf{0}$, or to find the global minimum of the nonlinear function $\phi(\boldsymbol{x})$. As a framework for our implementation of the IN/GB method, we use appropriately modified routines from the 
packages INTBIS (Kearfott et al., 1990) and INTLIB (Kearfott et al., 1994).

It should be emphasized that the enclosure, existence, and uniqueness properties discussed above, which are the basis of the IN/GB method, can be derived without making any strong assumptions about the function $\mathbf{f}(\boldsymbol{x})$ for which roots (zeros) are sought. The function must have a finite number of roots over the search interval of interest; however, no special properties such as convexity or monotonicity are required, and $\mathbf{f}(\boldsymbol{x})$ may have trancendental terms (e.g., Hua et al., 1998). It is assumed that $\mathbf{f}(\boldsymbol{x})$ is continuous over each interval being tested; however, it need not be continuously differentiable. Instead, as shown by Neumaier (1990), $\mathbf{f}(\boldsymbol{x})$ need only be Lipschitz continuous over the interval of interest; thus, functions with slope discontinuities can be handled. In order to apply the method, it must be possible to determine an interval extension of the Jacobian matrix (or of the "Lipschitz matrix" if $\mathbf{f}(\boldsymbol{x})$ is not continuously differentiable). In general, this requires having an analytic expression for $\mathbf{f}(\boldsymbol{x})$; thus, the interval approach is generally not suitable if $\mathbf{f}(\boldsymbol{x})$ is some kind of "black box" function. One difficulty with the interval-Newton approach is that if a solution occurs at a singular point (i.e., where the Jacobian of $\mathbf{f}(\boldsymbol{x})$ is singular), then it is not possible to obtain the result that identifies a unique solution. For such a case, the eventual result from the IN/GB algorithm will be a very narrow interval for which all that can be concluded is that it may contain one or more solutions. In other words, the algorithm will not miss the solution (so the guarantee to enclose all solutions remains), but rather, will enclose it within a narrow interval which can then be examined using an alternative methodology (e.g., Kearfott et al., 2000). This situation does not occur in any of the example problems considered here.

For improving the efficiency of IN/GB methods, there are various approaches, including: 1. Methods for dealing with the "dependency" issue in interval arithmetic, which may prevent the computation of interval extensions that tightly bound the function range (e.g. Ratscheck and Rokne, 1984; Makino and Berz, 1999; Jansson, 2000); 2. Techniques that involve changes to the methodology at the level of the nonlinear equation system (e.g., Alefeld et al., 1998; Granvilliers and Hains, 2000; van Hentenryck et al., 1997; Ratz, 1994; Herbort and Ratz, 1997; Yamamura et al., 1998; Yamamura and Nishizawa, 1999); 3. Methods that seek to make improvements in solving the linear interval system defined by Eq. (1), the interval-Newton equation (e.g., Kearfott, 1990; Hu, 1990; Kearfott et al., 1991; Gan et al., 1994; Hansen, 1997); or some combination of the above (e.g., Madan, 1990; Dinkel et al., 1991; Kearfott, 1991; Kearfott, 1997). A comprehensive review or these and other techniques is beyond the scope of this paper. Our initial focus here is on a methodology for solving the interval-Newton equation through the use of a hybrid preconditioning 
technique. This combines a standard inverse-midpoint preconditioner with strategies developed from the optimal preconditioning concepts of Kearfott and colleagues (Kearfott, 1990; Kearfott et al., 1991; Kearfott, 1996). Thus, we now provide some background on the solution of linear interval equation systems and on these optimal preconditioning concepts.

\subsection{Linear Interval Systems}

Consider a linear interval equation system $\mathbf{A} z=\mathbf{B}$, where the coefficient matrix $\mathbf{A}$ and the right-handside vector $\mathbf{B}$ are intervals. The solution set $S$ of this linear interval system is generally defined to be the set of all vectors $\mathbf{z}$ that satisfy the real linear system $\breve{A} \mathbf{z}=\mathbf{b}$, where $\breve{A}$ is any real matrix contained in $\mathbf{A}$ and $\mathbf{b}$ is any real vector contained in $\mathbf{B}$; that is, $S=\{\mathbf{z} \mid \breve{A} \mathbf{z}=\mathbf{b}, \breve{A} \in \mathbf{A}, \mathbf{b} \in \mathbf{B}\}$. However, as discussed and illustrated by Hansen (1992) and Kearfott (1996), this set is in general not an interval, and may have a very complex polygonal geometry. Thus to "solve" the linear system, one instead seeks an interval (solution hull) $\mathbf{Z}$ containing $S$. Computing the exact solution hull (tightest interval containing $S$ ) is NP-hard; however, it is generally relatively easy to obtain an interval $\mathbf{Z}$ that contains, but may overestimate, the exact solution hull. As discussed in detail by Kearfott (1996), the most common methods for doing this are direct elimination methods (e.g., interval Gaussian elimination), interval Gauss-Seidel (e.g., Hansen and Sengupta, 1981; Hansen and Greenburg, 1983), and the Krawczyk method (e.g., Krawczyk, 1969). For any of these methods, the use of some preconditioning technique is necessary in practice to obtain reasonably tight bounds on the solution set.

For the problem of interest, the interval-Newton equation, Eq. (1), the interval coefficient matrix is $\mathbf{A}=F^{\prime}\left(\mathbf{X}^{(k)}\right)$, the interval extension of the Jacobian over the current subinterval, the interval right-hand side is the degenerate (thin) interval $\mathbf{B}$ whose components are $B_{i}=\left[-f_{i}\left(\boldsymbol{x}^{(k)}\right),-f_{i}\left(\boldsymbol{x}^{(k)}\right)\right]$, and the solution vector is $\mathbf{N}^{(k)}-\boldsymbol{x}^{(k)}$. In the context of the interval-Newton method, the approach for solving the linear system (i.e. bounding the solution set) that is most attractive (Kearfott, 1996) and widely used is interval Gauss-Seidel, as described in more detail below.

\subsection{Interval Gauss-Seidel}

At the core of the interval-Newton method is the interval Gauss-Seidel procedure that is used to solve Eq. (1) for the image $\mathbf{N}^{(k)}$. The interval-Newton equation is first preconditioned using a real matrix $Y^{(k)}$. 
The preconditioned linear interval equation system can then be expressed as

$$
Y^{(k)} F^{\prime}\left(\mathbf{X}^{(k)}\right)\left(\mathbf{N}^{(k)}-\boldsymbol{x}^{(k)}\right)=-Y^{(k)} \mathbf{f}\left(\boldsymbol{x}^{(k)}\right)
$$

The preconditioner $Y^{(k)}$ used here is commonly taken to be an inverse-midpoint preconditioner (Hansen, 1965; Hansen and Sengupta, 1981; Hansen and Greenburg, 1983), which may be either the inverse of the real matrix formed from the midpoints of the elements of the interval Jacobian $F^{\prime}\left(\mathbf{X}^{(k)}\right)$, or the inverse of the real matrix determined by evaluating the point Jacobian $f^{\prime}(\boldsymbol{x})$ at the midpoint $\boldsymbol{x}^{(k)}$ of $\mathbf{X}^{(k)}$.

Defining $\mathbf{y}_{i}$ as the the $i$-th row of the preconditioning matrix, and $\mathbf{A}_{i}$ as the $i$-th column of the interval Jacobian $F^{\prime}\left(\mathbf{X}^{(k)}\right)$, then, beginning with $\mathbf{X}=\mathbf{X}^{(k)}$, the interval Gauss-Seidel scheme used in connection with interval-Newton methods proceeds component by component according to

$$
\begin{aligned}
N_{i} & =x_{i}-\frac{\mathbf{y}_{i} \mathbf{f}(\boldsymbol{x})+\sum_{\substack{j=1 \\
j \neq i}}^{n} \mathbf{y}_{i} \mathbf{A}_{j}\left(X_{j}-x_{j}\right)}{\mathbf{y}_{i} \mathbf{A}_{i}} \\
& =x_{i}-\frac{Q_{i}\left(\mathbf{y}_{i}\right)}{D_{i}\left(\mathbf{y}_{i}\right)},
\end{aligned}
$$

where $\mathbf{y}_{i} \mathbf{A}_{j}$ indicates the inner product of the real row vector $\mathbf{y}_{i}$ and the interval column vector $\mathbf{A}_{j}$, and $Q_{i}\left(\mathbf{y}_{i}\right)$ and $D_{i}\left(\mathbf{y}_{i}\right)$ are, respectively the numerator and denominator in the final term in Eq. (3), expressed as functions of $\mathbf{y}_{i}$, and then

$$
X_{i} \leftarrow X_{i} \cap N_{i}
$$

for $i=1, \ldots, n$. Note that after component $N_{i}$ of the image is calculated from Eq. (3) that the intersection in Eq. (4) is immediately performed, and the updated $X_{i}$ then used in the computation of subsequent components of the image. This means that this procedure actually does not enclose the full solution set of Eq. (1), but only the part of the solution set necessary for the interval-Newton iteration. Generally only one pass is made through Eqs. (3-4) and so after all the $X_{i}$ have been updated the result is $\mathbf{X}=\mathbf{X}^{(k+1)}$, the next interval-Newton iterate.

\subsection{Preconditioners}

The inverse-midpoint preconditioner is a good general-purpose preconditioner. However, as demonstrated by Kearfott (1990), it is not always the most effective approach. Thus, it is possible to seek other preconditioners that are optimal in some sense. The basic concepts in generating optimal preconditioners 
for the interval Gauss-Seidel step were pioneered by Kearfott and colleagues (e.g., Kearfott, 1990; Kearfott et al., 1991; Hu, 1990) and summarized in some detail by Kearfott (1996).

In these studies, a distinction is first made between contracting (C) and splitting (E) preconditioners. A preconditioning row $\mathbf{y}_{i}$ is called a C-preconditioner if $0 \notin D_{i}$. In this case, since the denominator in Eq. (3) does not contain zero, the resulting $N_{i}$ is a single connected interval. On the other hand, a preconditioning row $\mathbf{y}_{i}$ is called a E-preconditioner if $0 \in D_{i}, 0 \neq D_{i}$ and $0 \notin Q_{i}$. In this case, since the denominator in Eq. (3) contains zero, an extended interval arithmetic (Kahan-Novoa-Ratz arithmetic) is needed to compute $N_{i}$ and the result is the union of two disconnected semi-infinite intervals [see Kearfott (1996) for details and examples]. When used in the intersection step, Eq. (4), the resulting $X_{i}$ consists of two finite disconnected intervals, and so an E-preconditioner can serve as a tessellation scheme in addition to the usual tessellation done in the bisection step of the IN/GB algorithm.

For either C- or E-preconditioners, various optimality criteria can be defined, generally based on some property of $N_{i}$. In the context of interval-Newton, the most useful are typically the width-optimal preconditioner and the endpoint-optimal preconditioners. To determine a width-optimal preconditioner, elements of $\mathbf{y}_{i}$ are sought that minimize the width of $N_{i}$. To determine an endpoint-optimal preconditioner, elements of $\mathbf{y}_{i}$ are sought that either maximize the left endpoint (lower bound) or minimize the right endpoint (upper bound) of $N_{i}$. Kearfott (1990) showed that, under some mild assumptions, these optimization problems can be formulated and solved as linear programming (LP) problems. While the underlying optimization problems have $n$ degrees of freedom (the $n$ elements of $\mathbf{y}_{i}$ ), the corresponding LP problems have at least $4 n+2$ variables, as a number of auxiliary variables must be introduced in order to obtain the LP formulation. Computational experience (Kearfott, 1990) has shown that, on some problems, the use of the LP preconditioners can provide a significant reduction both in the number of subintervals that must be considered in the interval-Newton algorithm and in the overall CPU time required. However, in other problems, the overhead required to implement the LP-based preconditioning scheme outweighed any reduction in the number of subintervals tested, and overall CPU time actually increased. As one idea to make the optimal preconditioners more practical, Kearfott (1990) suggested considering a sparse preconditioner, that is, one in which only a small number of the elements of $\mathbf{y}_{i}$ are allowed to be nonzero. A special case of this, the properties of which have been described by Kearfott et al. (1991), is the "pivoting" preconditioner, in which there is only one nonzero element in $\mathbf{y}_{i}$. In the new hybrid preconditioning approach described below, the concept of the pivoting preconditioner is adopted. 


\section{Hybrid Preconditioning Approach}

We seek to develop an approach to preconditioning that results in a large reduction in the number of subintervals that must be tested in the interval-Newton algorithm, but that also can be implemented with little computational overhead, so that large savings in computation time can be realized. To do this we adopt a hybrid approach in which a simple pivoting preconditioner is used in combination with the standard inverse-midpoint scheme.

In a pivoting preconditioner, only one element of the preconditioning row $\mathbf{y}_{i}$ is nonzero, and it is assigned a value of one. Thus, if element $j$ in $\mathbf{y}_{i}$ is the "pivot element", then $y_{i j}=1$ and all the other elements of $\mathbf{y}_{i}$ are zero. In applying such a preconditioning row in Eq. (3), the results for $N_{i}$ will obviously depend on which element $j$ of $\mathbf{y}_{i}$ is used as the pivot. This can be expressed as

$$
\left(N_{i}\right)_{j}=x_{i}-\left(\frac{Q_{i}}{D_{i}}\right)_{j}
$$

where

$$
\left(Q_{i}\right)_{j}=f_{j}(\boldsymbol{x})+\sum_{\substack{k=1 \\ k \neq i}}^{n} A_{j k}\left(X_{k}-x_{k}\right)
$$

and

$$
\left(D_{i}\right)_{j}=A_{j i}
$$

Here and below, the notation $(\cdot)_{j}$ is used to indicate a quantity that has been evaluated using element $j$ of $\mathbf{y}_{i}$ as the pivot in the pivoting preconditioner. Clearly, the results obtained for the image component $N_{i}$, and thus the intersection $N_{i} \cap X_{i}$, can be manipulated by choosing different elements $j$ to be the pivot. Thus, some optimality criteria are needed to decide which element $j$ to choose.

In order to reduce the number of subintervals that must be tested during the interval-Newton algorithm, an obvious goal is to reduce the number of bisections that occur. Bisections do not occur whenever $\mathbf{X}^{(k)} \cap \mathbf{N}^{(k)}=\emptyset$, meaning there is no root in the current subinterval $\mathbf{X}^{(k)}$ and it can be discarded, or when $\mathbf{N}^{(k)} \subset \mathbf{X}^{(k)}$, meaning that there is known to be a unique root in the current subinterval $\mathbf{X}^{(k)}$, or if the intersection $\mathbf{X}^{(k)} \cap \mathbf{N}^{(k)}$ is sufficiently smaller than $\mathbf{X}^{(k)}$, meaning that another interval-Newton test is tried on $\mathbf{X}^{(k)} \cap \mathbf{N}^{(k)}$ rather than bisecting it. An optimality criterion that increases the likelihood of all these possibilities is to seek to use a preconditioning row $\mathbf{y}_{i}$ that minimizes the width of $N_{i} \cap X_{i}$. This is treated as two separate cases: 
1. The row $\mathbf{y}_{i}$ is a "discarding-optimal" preconditioning row provided it results in $N_{i} \cap X_{i}=\emptyset$, indicating that $N_{i} \cap X_{i}$ has a minimum width of zero.

2. The row $\mathbf{y}_{i}$ is a "contraction-optimal" preconditioning row provided that $N_{i} \cap X_{i}$ is nonempty, and that it minimizes $w\left[N_{i} \cap X_{i}\right]$, the width of $N_{i} \cap X_{i}$. Note that this is somewhat different than the more common width-optimal preconditioner that minimizes the width of $N_{i}$.

We will seek to find optimal pivoting preconditioners of these types. A pivoting preconditioner row $\mathbf{y}_{i}$ is discarding-optimal if there is some pivot element $j$ in $\mathbf{y}_{i}$ for which $\left(N_{i}\right)_{j} \cap X_{i}=\emptyset$. A pivoting preconditioner row $\mathbf{y}_{i}$ is contraction-optimal when the pivot element $j$ is the solution to the optimization problem $\min _{j} w\left[\left(N_{i}\right)_{j} \cap X_{i}\right]$. If there exists a discarding-optimal row $\mathbf{y}_{i}$, then there is no contraction-optimal row.

To look for a discarding-optimal pivoting preconditioner row, and, if none exists, to determine the contraction-optimal pivoting preconditioner row, it is necessary to compute the endpoints of $\left(N_{i}\right)_{j}$ using Eq. (5-7). Since these computations are done only for the purpose of selecting a preconditioning row, they can be done cheaply using real (not interval) arithmetic. (Once the preconditioning row is chosen it must be implemented in Eq. (3) using interval arithmetic.) Two cases must be considered, corresponding to the Cand E-type preconditioners. If $0 \notin A_{j i}$, then we refer to element $j$ as a "C-pivot"; the right endpoint (upper bound) of $\left(N_{i}\right)_{j}$ is then given by

$$
R\left[\left(N_{i}\right)_{j}\right]=x_{i}-\min \left\{\frac{\left(Q_{i}\right)_{j}}{\underline{A_{j i}}}, \frac{\left(Q_{i}\right)_{j}}{\overline{A_{j i}}}, \frac{\overline{\left(Q_{i}\right)_{j}}}{\underline{A_{j i}}}, \frac{\overline{\left(Q_{i}\right)_{j}}}{\overline{A_{j i}}}\right\}
$$

and the left endpoint (lower bound) by

$$
L\left[\left(N_{i}\right)_{j}\right]=x_{i}-\max \left\{\frac{\left(Q_{i}\right)_{j}}{\underline{A_{j i}}}, \frac{\left(Q_{i}\right)_{j}}{\overline{A_{j i}}}, \frac{\overline{\left(Q_{i}\right)_{j}}}{\underline{A_{j i}}}, \frac{\overline{\left(Q_{i}\right)_{j}}}{\overline{A_{j i}}}\right\} .
$$

If $0 \in A_{j i}$ and $0 \notin\left(Q_{i}\right)_{j}$, then we refer to element $j$ as an "E-pivot"; for this case, Kahan-NovoaRatz arithmetic is used, and $\left(N_{i}\right)_{j}=\left(N_{i}\right)_{j}^{-} \cup\left(N_{i}\right)_{j}^{+}$, the union of the semi-infinite intervals $\left(N_{i}\right)_{j}^{-}=$ $\left[-\infty, R\left[\left(N_{i}\right)_{j}^{-}\right]\right]$and $\left(N_{i}\right)_{j}^{+}=\left[L\left[\left(N_{i}\right)_{j}^{+}\right], \infty\right]$, where for the case $\overline{\left(Q_{i}\right)_{j}}<0$, the finite bounds on $\left(N_{i}\right)_{j}^{-}$ and $\left(N_{i}\right)_{j}^{+}$are given by

$$
\begin{aligned}
& R\left[\left(N_{i}\right)_{j}^{-}\right]=x_{i}-\frac{\overline{\left(Q_{i}\right)_{j}}}{\overline{A_{j i}}} \\
& L\left[\left(N_{i}\right)_{j}^{+}\right]=x_{i}-\frac{\overline{\left(Q_{i}\right)_{j}}}{\overline{A_{j i}}},
\end{aligned}
$$

and, for $\underline{\left(Q_{i}\right)_{j}}>0$, by

$$
R\left[\left(N_{i}\right)_{j}^{-}\right]=x_{i}-\frac{\left(Q_{i}\right)_{j}}{\overline{A_{j i}}}
$$




$$
L\left[\left(N_{i}\right)_{j}^{+}\right]=x_{i}-\frac{\left(Q_{i}\right)_{j}}{\underline{A_{j i}}} .
$$

If $0 \in A_{j i}$ and $0 \in\left(Q_{i}\right)_{j}$, then there is no pivoting preconditioner corresponding to the element $j$.

As already noted, use of the inverse-midpoint preconditioner does not always result in good performance for the interval-Newton algorithm. In our experience, the use of pivoting preconditioners alone also does not always result in good performance for the interval-Newton algorithm. Thus, we have adopted a hybrid strategy. The basic idea is that, in determining a preconditioning row $\mathbf{y}_{i}$, we first search for a discardingoptimal pivoting preconditioner (since the vast majority of the subintervals that need to be tested in the interval-Newton procedure will contain no roots and need to be discarded) and while doing so also determine the information needed to pick a contraction-optimal pivoting preconditioner if no discarding-optimal pivoting preconditioner exists. If in fact there is no discarding-optimal pivoting preconditioner, then either the contraction-optimal pivoting preconditioner or the inverse-midpoint preconditioner is used, depended on which yields the smaller result for the width $w\left[N_{i} \cap X_{i}\right]$.

This hybrid scheme for determining the preconditioning row $\mathbf{y}_{i}$ can be summarized algorithmically as follows:

1. Initialize: Set $j=1, j^{*}=0$ and $w^{*}$ to some arbitrarily large number ( $j^{*}$ and $w^{*}$ will be used to store information for determining a contraction-optimal pivoting preconditioner).

2. Determine endpoints: Compute $\left(Q_{i}\right)_{j}$ and using this and $A_{j i}$ determine whether $j$ is either a C- or E-type pivot. If $j$ is a C-type pivot then determine the endpoints of $\left(N_{i}\right)_{j}$ from Eqs. (8-9). If $j$ is an E-type pivot then determine the finite endpoints of $\left(N_{i}\right)_{j}$ from Eqs. (10-13). If $j$ is neither C- or E-type then go to Step 5.

3. Check if discarding-optimal: If $j$ is C-type and either $R\left[\left(N_{i}\right)_{j}\right]<\underline{X_{i}}$ or $L\left[\left(N_{i}\right)_{j}\right]>\overline{X_{i}}$, or if $j$ is E-type and both $L\left[\left(N_{i}\right)_{j}\right]>\overline{X_{i}}$ and $R\left[\left(N_{i}\right)_{j}\right]<\underline{X_{i}}$, then $\left(N_{i}\right)_{j} \cap X_{i}=\emptyset$ and a discarding-optimal pivoting preconditioner has been found. Stop and return to the interval Gauss-Seidel computation, Eq. (3), using this as the preconditioning row. This should result in the current subinterval $\mathbf{X}$ being discarded. If $j$ is not discarding-optimal, then continue.

4. Update minimum intersection width: Compute $w\left[\left(N_{i}\right)_{j} \cap X_{i}\right]$, noting that if $j$ is E-type that this is the sum of the widths of $\left[\left(N_{i}\right)_{j}^{-} \cap X_{i}\right]$ and $\left[\left(N_{i}\right)_{j}^{+} \cap X_{i}\right]$. If $w\left[\left(N_{i}\right)_{j} \cap X_{i}\right]<w^{*}$, then $j^{*} \leftarrow j$ and $w^{*} \leftarrow w\left[\left(N_{i}\right)_{j} \cap X_{i}\right]$. 
5. Iterate: If $j<n$, then set $j \leftarrow j+1$ and return to Step 2. If $j=n$, continue.

6. Using $\mathbf{y}_{i}^{\text {inv }}$, the $i$-th row of the inverse-midpoint preconditioner, compute from Eq. (3) the corresponding image element $N_{i}^{\text {inv }}$ and then $w^{\text {inv }}=w\left[N_{i}^{\text {inv }} \cap X_{i}\right]$. If $w^{\text {inv }} \leq w^{*}$, then use $\mathbf{y}_{i}^{\text {inv }}$ as the preconditioning row. If $w^{*}<w^{\text {inv }}$, then use the pivoting precondition with $j=j^{*}$, since it is contraction-optimal.

The procedure outlined above can be implemented very efficiently using real (not interval) arithmetic, since it is used only to select a preconditioning row. When the preconditioning row selected is returned to Eq. (3), the computation is done rigorously using interval arithmetic. The performance of this hybrid preconditioning scheme will be demonstrated using the test problems below.

\section{Selection of Real Point}

In the interval-Newton equation, Eq. (1), the real point $\boldsymbol{x}^{(k)}$ is typically chosen to be the midpoint of the current subinterval $\mathbf{X}^{(k)}$. However, in principle $\boldsymbol{x}^{(k)}$ can be any point in $\mathbf{X}^{(k)}$, and so could be chosen to try to improve the performance of the interval-Newton procedure. For example, Alefeld et al. (1998) suggest a scheme for selecting an improved real point in the context of a univariate interval-Newton algorithm. This scheme is based on use of a local equation solver to make the function value at the real point closer to zero. We use a different type of scheme here; it is coupled to the preconditioning scheme discussed above and tries to further reduce the width of the intersection $\left(N_{i}\right)_{j} \cap X_{i}$ determined using a pivoting preconditioner row $\mathbf{y}_{i}$ with pivot element $j$. This scheme could be applied in connection with both the C-and E-preconditioner cases, but the implementation for the C-preconditioner case involves less computational overhead than in the E-preconditioner case, and so we have chosen to apply it only when $j$ is a C-pivot.

In order to reduce $w\left[\left(N_{i}\right)_{j} \cap X_{i}\right]$, we can manipulate the real point $\boldsymbol{x}$ in Eqs. (5-6) to try to either minimize the right end point $R\left[\left(N_{i}\right)_{j}\right]$ or maximize the left end point $L\left[\left(N_{i}\right)_{j}\right]$. In our approach, we try both of these possibilities and then use the one that provides the largest reduction in $w\left[\left(N_{i}\right)_{j} \cap X_{i}\right]$. To determine exactly the optimal endpoints of $\left(N_{i}\right)_{j}$ with respect to the real point $\boldsymbol{x}$ would require optimizing an expression involving the possibly nonlinear function $f_{j}(\boldsymbol{x})$. Since this could be quite difficult, we will seek only improved, not optimal, values of the endpoints. For this purpose, we will treat $f_{j}$ as depending only on $x_{i}$, the current coordinate in the Gauss-Seidel procedure.

To see how the real point $\boldsymbol{x}$ can be adjusted to improve the endpoints of $\left(N_{i}\right)_{j}$ it is convenient to rewrite 
Eq. (5-7) as

$$
\left(N_{i}\right)_{j}=x_{i}-\frac{\left(Q_{i}\right)_{j}}{A_{j i}}=x_{i}-\frac{f_{j}\left(x_{i}\right)+H}{A_{j i}}
$$

where $H$ is the summation

$$
H=\sum_{\substack{k=1 \\ k \neq i}}^{n} A_{j k}\left(X_{k}-x_{k}\right) .
$$

The non-current coordinates $x_{k}, k \neq i$, appear only in the term $H / A_{j i}$. Thus to determine how to select values of the non-current coordinates in the real point, we need only consider the effect of this last term on the endpoints of $\left(N_{i}\right)_{j}$. To try to best improve (increase) $L\left[\left(N_{i}\right)_{j}\right]$, the values of $x_{k}, k \neq i$, that result in the minimum upper bound for $H$ should be sought if $\underline{A_{j i}}>0$, and the values of $x_{k}, k \neq i$, that result in the maximum lower bound for $H$ should be sought if $\overline{A_{j i}}<0$. Similarly, to try to best improve (decrease) $R\left[\left(N_{i}\right)_{j}\right]$, the values of $x_{k}, k \neq i$, that result in the maximum lower bound for $H$ should be sought if $\underline{A_{j i}}>0$, and the values of $x_{k}, k \neq i$, that result in the minimum upper bound for $H$ should be sought if $\overline{A_{j i}}<0$.

Using Eq. (15), and remembering that $x_{k} \in X_{k}$, it can be determined that the values of $x_{k}, k \neq i$, that minimize $\bar{H}$ are given by

$$
x_{k}=x_{k}^{+}=\left\{\begin{array}{rll}
\overline{X_{k}} & \text { if } & \frac{A_{j k}}{2}>0 \\
\frac{X_{k}}{\left.A_{j k}\left(\overline{X_{k}}\right)-\underline{A_{j k}} \underline{X_{k}}\right)} & \text { if } & \overline{A_{j k}}<0 \\
\overline{X_{k}}-\underline{X_{k}} & \text { if } & 0 \in A_{j k}
\end{array}\right.
$$

and that the values of $x_{k}, k \neq i$, that maximize $\underline{H}$ are given by

$$
x_{k}=x_{k}^{-}=\left\{\begin{array}{rll}
\frac{X_{k}}{\overline{X_{k}}} & \text { if } & \frac{A_{j k}}{A_{j k}}<0 \\
\frac{A_{j k}\left(\frac{X_{k}}{\bar{X}_{k}}-\underline{A_{j k}}\left(\overline{X_{k}}\right)\right.}{X_{k}} & \text { if } & 0 \in A_{j k} .
\end{array}\right.
$$

Thus, to try to increase $L\left[\left(N_{i}\right)_{j}\right]$, then, for $k \neq i$, choose $x_{k}=x_{k}^{+}$if $\underline{A_{j i}}>0$ and $x_{k}=x_{k}^{-}$if $\overline{A_{j i}}<0$, and to try to decrease $R\left[\left(N_{i}\right)_{j}\right]$, then, for $k \neq i$, choose $x_{k}=x_{k}^{-}$if $\underline{A_{j i}}>0$ and $x_{k}=x_{k}^{+}$if $\overline{A_{j i}}<0$. Note that these choices will not necessarily have the desired effect, since the dependence of $f_{j}$ on $x_{k}, k \neq i$, has been neglected. Nevertheless, we have found this to be a useful scheme for many problems.

Selection of a value of the current coordinate $x_{i}$ to increase $L\left[\left(N_{i}\right)_{j}\right]$ or decrease $R\left[\left(N_{i}\right)_{j}\right]$ is less straightforward, since, depending on whether the choice is made based on the $x_{i}$ or the $f_{j}\left(x_{i}\right) / A_{j i}$ term in Eq. (14), the choice will be different and contradictory. Looking just at the $x_{i}$ term, $L\left[\left(N_{i}\right)_{j}\right]$ can be increased the 
most by setting $x_{i}=\overline{X_{i}}$, and $R\left[\left(N_{i}\right)_{j}\right]$ can be decreased the most by setting $x_{i}=\underline{X_{i}}$. Looking just at the $f_{j}\left(x_{i}\right) / A_{j i}$ term, we first note that $A_{j i}$ is an interval extension of $\partial f_{j} / \partial x_{i}$, so that if $\underline{A_{j i}}>0$, then $f_{j}$ is monotonically increasing with respect to $x_{i}$, and if $\overline{A_{j i}}<0$, then $f_{j}$ is monotonically decreasing with respect to $x_{i}$ (the case $0 \in A_{j i}$ is not considered since we are working with C-pivots only). Thus, in either the case of $\underline{A_{j i}}>0$ or $\overline{A_{j i}}<0$, the term $f_{j}\left(x_{i}\right) / A_{j i}$ has its maximum lower bound at $x_{i}=\overline{X_{i}}$ and its minimum upper bound at $x_{i}=\underline{X_{i}}$. So, based on the effect of the $f_{j}\left(x_{i}\right) / A_{j i}$ term, $L\left[\left(N_{i}\right)_{j}\right]$ can be increased the most by setting $x_{i}=\underline{X_{i}}$, and $R\left[\left(N_{i}\right)_{j}\right]$ can be decreased the most by setting $x_{i}=\overline{X_{i}}$, which is just the opposite of the conclusion based on the $x_{i}$ term. In order to keep computational overhead low, a simple heuristic is used to make the choice between these two possibilities. The heuristic is that the choice of $x_{i}$ is based on the $f_{j}\left(x_{i}\right) / A_{j i}$ term whenever it is possible that this will result in $0 \notin\left(Q_{i}\right)_{j}$. Otherwise, the choice of $x_{i}$ is based on the $x_{i}$ term.

To understand this heuristic and see how it is implemented, it should first be noted that in order to demonstrate that the subinterval currently being tested can be discarded, it is necessary that $0 \notin\left(Q_{i}\right)_{j}$. If this were not the case then, from Eq. (14), $x_{i} \in\left(N_{i}\right)_{j}$, meaning that the intersection $\left(N_{i}\right)_{j} \cap X_{i}$ is nonnull and thus the current interval cannot be discarded. Thus, it is desirable that $0 \notin\left(Q_{i}\right)_{j}$. However, it should next be noted that, in $\left(Q_{i}\right)_{j}=f_{j}+H$, the summation term $H$ from Eq. (15) does include zero since $x_{k} \in X_{k}$. Thus, the result $0 \notin\left(Q_{i}\right)_{j}$ can only be obtained if there is a positive value of $f_{j}$ whose magnitude is large enough so that $\underline{H}+f_{j}>0$, or there is a negative value of $f_{j}$ whose magnitude is large enough so that $\bar{H}+f_{j}<0$. Since an interval extension $F_{j}$ of $f_{j}$ has already been determined as part of the function range test, and it is known that $0 \in F_{j}$ since otherwise the current subinterval would have already been discarded, these possibilities can be easily checked. If $\underline{H}+\overline{F_{j}}>0$ or if $\bar{H}+\underline{F_{j}}<0$, then it is possible, by choosing $x_{i}$ based on the $f_{j}\left(x_{i}\right) / A_{j i}$ term, that the situation $0 \notin\left(Q_{i}\right)_{j}$ could be achieved. Thus, to try to increase $L\left[\left(N_{i}\right)_{j}\right]$, set $x_{i}=\underline{X_{i}}$ if $\underline{H}+\overline{F_{j}}>0$, but otherwise set $x_{i}=\overline{X_{i}}$. Similarly, to try to decrease $R\left[\left(N_{i}\right)_{j}\right]$, set $x_{i}=\overline{X_{i}}$ if $\bar{H}+\underline{F_{j}}<0$, but otherwise set $x_{i}=\underline{X_{i}}$.

Because the strategy for adjusting the real point can result in a significant computational overhead, especially when function evaluations are expensive, it is only attempted when there is reason to believe that it might be effective. To make the decision on whether or not to apply the real point selection scheme, we use two heuristics based on our experience with the approach. The first heuristic comes from the observation that if the pivoting preconditioner has resulted in some improvement, then the real point selection scheme is often effective in providing additional improvement. Thus, we apply the real point selection strategy 
whenever, after Step 4 above, $\left\{w\left[X_{i}\right]-w\left[\left(N_{i}\right)_{j} \cap X_{i}\right]\right\} / w\left[X_{i}\right]>\epsilon_{1}$, where $\epsilon_{1}$ indicates some minimum level of improvement. Currently we use $\epsilon_{1}=0$, so if there is any improvement we proceed to apply the real point selection scheme. The second heuristic is based on the observation that, even if $\left(N_{i}\right)_{j} \supset X_{i}$, some improvements may still be possible if the endpoints of $\left(N_{i}\right)_{j}$ are relatively close to the corresponding endpoints of $X_{i}$. Thus, we also apply the real point selection scheme whenever, after Step 4 above, $\left\{\left[\underline{X_{i}}-\underline{\left.\left(N_{i}\right)_{j}\right]}+\left[\overline{\left(N_{i}\right)_{j}}-\overline{X_{i}}\right]\right\} /\left[\overline{X_{i}}-\underline{X_{i}}\right]=\left\{w\left[\left(N_{i}\right)_{j}\right]-w\left[X_{i}\right]\right\} / w\left[X_{i}\right]<\epsilon_{2}\right.$, where we currently use $\epsilon_{2}=0.1$. This requires that both endpoints of $\left(N_{i}\right)_{j}$ be relatively close to the corresponding endpoints of $X_{i}$. One might also use a heuristic requiring that only one endpoint of $\left(N_{i}\right)_{j}$ be close to the corresponding endpoint of $X_{i}$, but this has not been tried. If neither of these two heuristic conditions is satisfied, then the real point selection scheme is skipped for the current pivot $j$.

The scheme used to select a real point $\boldsymbol{x} \in \mathbf{X}$ is summarized algorithmically below. Note that this procedure is coupled to the preconditioning scheme, and is implemented immediately after Step 4 in the scheme outlined above.

\subsection{Check for improved real point:}

(a) (Check whether to skip) Set $\epsilon_{1}$ and $\epsilon_{2}$ (we use $\epsilon_{1}=0$ and $\epsilon_{2}=0.1$ ).

i. If $0 \in A_{j i}$, that is if $j$ is E-type, go to Step 5 .

ii. If $\left\{w\left[X_{i}\right]-w\left[\left(N_{i}\right)_{j} \cap X_{i}\right]\right\} / w\left[X_{i}\right]>\epsilon_{1}$, go to Step 4.1(b).

iii. If $\left\{w\left[\left(N_{i}\right)_{j}\right]-w\left[X_{i}\right]\right\} / w\left[X_{i}\right]<\epsilon_{2}$, then continue; otherwise, go to Step 5 .

(b) (Check left endpoint) Compute $w\left[\left(N_{i}\right)_{j} \cap X_{i}\right]$ using a trial real point $\hat{\boldsymbol{x}}$, where $\hat{x}_{k}=x^{+}, k \neq i$, if $\underline{A_{j i}}>0$, or $\hat{x}_{k}=x^{-}, k \neq i$, if $\overline{A_{j i}}<0$, and using $\hat{x}_{i}=\underline{X_{i}}$ if $\underline{H}+\overline{F_{j}}>0$ or using $\hat{x}_{i}=\overline{X_{i}}$ otherwise. If $w\left[\left(N_{i}\right)_{j} \cap X_{i}\right]<w^{*}$, then $\boldsymbol{x} \leftarrow \hat{\boldsymbol{x}}, j^{*} \leftarrow j$, and $w^{*} \leftarrow w\left[\left(N_{i}\right)_{j} \cap X_{i}\right]$.

(c) (Check right endpoint) Compute $w\left[\left(N_{i}\right)_{j} \cap X_{i}\right]$ using a trial real point $\hat{\boldsymbol{x}}$, where $\hat{x}_{k}=x^{-}, k \neq i$, if $\underline{A_{j i}}>0$, or $\hat{x}_{k}=x^{+}, k \neq i$, if $\overline{A_{j i}}<0$, and using $\hat{x}_{i}=\overline{X_{i}}$ if $\bar{H}+\underline{F_{j}}<0$ or using $\hat{x}_{i}=\underline{X_{i}}$ otherwise. If $w\left[\left(N_{i}\right)_{j} \cap X_{i}\right]<w^{*}$, then $\boldsymbol{x} \leftarrow \hat{\boldsymbol{x}}, j^{*} \leftarrow j$, and $w^{*} \leftarrow w\left[\left(N_{i}\right)_{j} \cap X_{i}\right]$.

Note that if no change is made in the real point $\boldsymbol{x}$ in either of Steps 4.1(b) or 4.1(c) above, then $\boldsymbol{x}$ remains the midpoint of $\mathbf{X}$. As in the case of the preconditioning scheme, the steps outlined above can be implemented using real arithmetic. However, once the appropriate real point has been selected, the computation in Eq. (3) must be done using interval arithmetic. Also note that, if a component $x_{k}$ of the real point is selected to be equal to an endpoint of the current subinterval, then before substitution into Eq. (3), a directed rounding 
is done in order to ensure that $x_{k} \in X_{k}$. For example, if we set $x_{k}=\underline{X_{k}}$, then $x_{k}$ is rounded up, and if we set $x_{k}=\overline{X_{k}}$, then $x_{k}$ is rounded down.

In the usual interval-Newton approach, if $N_{i} \subset X_{i}$ for all components $i$ in the Gauss-Seidel solution of Eq. (1), then the conclusion is that there is a unique root in the current subinterval $\mathbf{X}$. This same conclusion is also valid when the preconditioning scheme described above is used, since this just means that a different preconditioning matrix is used in solving for each different component in the Gauss-Seidel scheme. However, this conclusion is in general not valid when the real point $\boldsymbol{x}$ is changed, as suggested above, with each different component in the Gauss-Seidel procedure, since in this case a different formulation of the intervalNewton equation is being used to compute each different component $N_{i}$ of the image. Nevertheless, since each $N_{i}$ is computed from a valid form of the interval-Newton equation, it is still possible to conclude that if there is a root whose $i$-th component $x_{i}^{*}$ is in $X_{i}$ then $x_{i}^{*}$ must be in $N_{i}$, and thus the use of the intersection $X_{i} \cap N_{i}$ to narrow or discard the current subinterval is still valid. If for some subinterval in which the real point is changed, and the result $N_{i} \subset X_{i}$ for all $i$ is obtained, then to try to show that this subinterval contains a unique root, we simply re-test this subinterval without use of the real point selection scheme described here.

We now consider several example problems in which the performance of the interval-Newton method with and without the new preconditioning and real point selection strategies is tested.

\section{Numerical Experiments and Results}

In this section, we present the results of numerical experiments done to test the effectiveness of the new hybrid preconditioning strategy and real point selection scheme described above. The test problems include a variety of global optimization and nonlinear equation solving problems arising in the context of process systems engineering. For each case, all problem information, including detailed equation formulations, equation parameters and other data, and the initial intervals used, are given in the references cited or in the discussion below. All computations were performed on a Sun Ultra 2/1300 workstation.

\subsection{Problem 1: Phase Stability Analysis}

This problem involves the determination of phase stability from the UNIQUAC model of excess Gibbs free energy (Tessier et al., 2000). The determination of phase stability is usually approached by using tangent plane analysis, which states that a phase (or "feed") at a specified temperature $T$, pressure $P$, and 
composition (mole fraction) $\mathbf{z}$ is not stable if the molar Gibbs energy vs. composition surface $g(\boldsymbol{x})$ ever falls below a plane tangent to the surface at $\mathbf{z}$. That is, the feed is not stable if the tangent plane distance

$$
D=g(\boldsymbol{x})-g_{0}-\sum_{i=1}^{n}\left(\frac{\partial g}{\partial x_{i}}\right)_{0}\left(x_{i}-z_{i}\right)
$$

is negative for any composition $\boldsymbol{x}$. Here the subscript zero indicates evaluation at $\boldsymbol{x}=\mathbf{z}$ and $n$ is the number of components. To determine whether $D$ is ever negative, an optimization problem is solved in which $D$ is minimized subject to $1-\sum_{i=1}^{n} x_{i}=0$. The stationary points in this optimization problem can be determined by solving the nonlinear equation system

$$
\begin{gathered}
{\left[\left(\frac{\partial g}{\partial x_{i}}\right)-\left(\frac{\partial g}{\partial x_{n}}\right)\right]-\left[\left(\frac{\partial g}{\partial x_{i}}\right)-\left(\frac{\partial g}{\partial x_{n}}\right)\right]_{0}=0, \quad i=1, \ldots, n-1} \\
1-\sum_{i=1}^{n} x_{i}=0 .
\end{gathered}
$$

This $n \times n$ equation system is used here as an example problem to test the new interval methodologies. For the case in which $g(\boldsymbol{x})$ is obtained using UNIQUAC, the details of the problem formulation are given by Tessier et al. (2000), who solve the problem using an interval-Newton approach.

The specific problems considered are: 1. A four-component system of acetic acid, benzene, furfural and cyclohexane 2. A five-component system containing the previous four components plus water. 3. A six-component system of benzene, cyclohexane, 1,2-ethanediol, furfural, heptane, and water. The first two problems were used by Tessier et al. (2000); the last is a new problem, with UNIQUAC parameters from Sørensen and Arlt (1979-1987). For each system, the UNIQUAC model was used and and a number of different feed compositions were considered. For solving these problems we use the methodology described by Tessier at al. (2000) for computing function extensions. This involves the use of monotonicity information and the evaluation of functions in the constrained space for which the mole fractions sum to one. Because this constrained-space extension requires that the components of the real point in the interval-Newton equations sum to one, we are unable to test the new real point selection scheme on this problem. Only the effect of the new hybrid preconditioning strategy will be investigated. The initial intervals used in all cases is $x_{i} \in[0,1], i=1, \ldots, n$.

All the problems considered were successfully solved, with the computational performance shown in Tables 1-3. In these Tables, computational performance is indicated both by the number of subintervals that needed to be considered in each case, as well as by the CPU time. Here "IMP" refers to the results for the 
inverse-midpoint preconditioner, "HP" refers to the results for the new hybrid preconditioner, and "ImpFac" refers to the improvement factor by which HP is better than IMP.

Looking first at the results for the four-component problem (Table 1), it is seen that the use of the hybrid preconditioning scheme has resulted in an order of magnitude reduction in the number of subintervals that needed to be considered in the interval-Newton algorithm. This is seen in the CPU time results as well, though these improvement factors are not as large as in the number of subintervals, reflecting the computational overhead required to implement the hybrid preconditioner. Based on an average over all the tested feeds, the improvement factor due to use of the hybrid preconditioner is about 10.9 based on number of subintervals and about 8.1 based on CPU time.

Not unexpectedly, improvement factors on the five- and six-component problems (Tables 2 and 3) are even more pronounced, since there is increasingly more to gain on the larger problems. For the fivecomponent problem the average improvement factor is about 16.6 based on number of subintervals and about 11.3 based on CPU time, while for the six-component problem the average improvement factors are about 590 based on number of subintervals and about 425 based on CPU time. For the third feed considered in the six-component problem, an improvement of three orders of magnitude was achieved in CPU time. A problem that originally took over 40 hours to solve, could be solved in less than two-and-a-half minutes when the new hybrid preconditioning strategy was used.

\subsection{Problem 2: Mixture Critical Points}

This problem involves the computation of mixture critical points from cubic equation-of-state models. Stradi et al. (2001) have recently described how this can be accomplished reliably using an interval-Newton methodology. In the problem formulation used by Stradi et al. (2001), the nonlinear equation system that must be solved is

$$
\begin{gathered}
\sum_{j=1}^{C} A_{i j} \Delta n_{j}=0, \quad i=1, \ldots, C \\
\sum_{i=1}^{C} \sum_{j=1}^{C} \sum_{k=1}^{C} A_{i j k} \Delta n_{i} \Delta n_{j} \Delta n_{k}=0 \\
1-\sum_{i=1}^{C} \Delta\left(n_{i}\right)^{2}=0,
\end{gathered}
$$

where $A_{i j}$ and $A_{i j k}$ represent the second and third order derivatives of the Helmholtz free energy $A$ with respect to composition, and are nonlinear functions of temperature $T$ and volume $V$, both of which are 
unknown. Also, $C$ is the total number of components and the $\Delta n_{i}, i=1, \ldots, C$, represent unknown changes in the number of moles of the $C$ components. This is a $(C+2) \times(C+2)$ equation system which when solved for $T, V$, and $\Delta n_{i}, i=1, \ldots, C$, yields the critical temperature and volume. For the cases considered here, $A(T, V)$ is determined using the Peng-Robinson equation-of-state model. Details of the problem formulation for this model, as well as model parameters and initial intervals used, are given by Stradi (1999) and Stradi et al. (2001).

Three specific test problems are considered: 1. A three-component mixture of methane(1), carbon dioxide(2), and hydrogen sulfide(3) with composition $x_{1}=0.0700, x_{2}=0.6160$, and $x_{3}=0.3140 ; 2$. A four-component mixture of methane(1), ethane(2), propane(3), and nitrogen(4) with composition $x_{1}=$ 0.4345, $x_{2}=0.0835, x_{3}=0.4330$, and $x_{4}=0.0490 ; 3$. A five-component mixture of methane(1), ethane(2), propane(3), $n$-butane(4) and nitrogen(5) with composition $x_{1}=0.9450, x_{2}=0.0260, x_{3}=$ $0.0081, x_{4}=0.0052$, and $x_{5}=0.0160$. For these problems, the effects of using both the new hybrid preconditioning strategy and the new real point selection scheme are considered.

All the problems were successfully solved, with the computational performance shown in Table 4 . Here the notation is the same as above, but with the additional notation "HP/RP" to indicate use of both the hybrid preconditioner and real point selection schemes. From these results, it can be seen first of all that use of the real point selection scheme does have a beneficial effect, both on the number of subintervals and the CPU time. The overall improvements, however, are much less dramatic than in the phase stability problem, and, also in contrast to the phase stability problem, the improvement factors decrease with increased problem size. This behavior suggests that, for these problems, the dominant factor leading to computational inefficiency is not in formulating or solving the interval-Newton equation, the factors addressed here, but instead in obtaining reasonably tight bounds on the function and Jacobian element ranges when their interval extensions are computed, a factor not addressed here. This overestimation in computing interval extensions is due to the dependency problem, which may arise when a variable occurs more than once in a function expression, and the interval extension is computed used interval arithmetic (the "natural" interval extension). While a variable may take on any value within its interval, it must take on the same value each time it occurs in an expression. However, this type of dependency is not recognized when the natural interval extension is computed. In effect, when the natural interval extension is used, the range computed for the function is the range that would occur if each instance of a particular variable were allowed to take on a different value in its interval range. The expressions for the functions and Jacobian elements for the critical point problem 
are quite complicated, and grow increasingly so as the number of components increases, and so it is particularly difficult to get tight interval extensions using interval arithmetic. For the phase stability problem, tighter interval extensions could be obtained by using the monotonicity and constrained-space extensions described by Tessier et al. (2000). Similar techniques might be useful in improving the performance of the interval-Newton algorithm on the mixture critical point problem.

\subsection{Problem 3: Parameter Estimation in VLE Modeling}

This is a problem that has also been used by Kim et al. (1990) and Esposito and Floudas (1998). It is a parameter estimation problem using the error-in-variable (EIV) approach to estimate parameters in the van Laar equation for activity coefficients used to model vapor-liquid equilibrium (VLE). These two parameters are estimated from binary VLE data for the binary system of methanol and 1,2-dichloroethane. The experimental data consist of five experimental data points for four measured state variables, namely pressure, temperature, liquid-phase mole fraction of methanol, and vapor-phase mole fraction of methanol. Complete details of the problem are given by Gau and Stadtherr (2000b), who formulate it as an unconstrained global optimization problem with 12 variables. A maximum-likelihood-based estimator is optimized by applying the interval-Newton methodology to solve for the stationary points in the optimization problem. The effects of using both the new hybrid preconditioning strategy and the new real point selection scheme are considered.

This global optimization problem was solved successfully, with computational performance results shown in Table 5. When using the standard inverse-midpoint preconditioner, the program was still running after two CPU days and was terminated at this point. However, when using the new hybrid preconditioner, the problem was solved in only 1504.2 CPU seconds. When the real point selection scheme was also applied, the CPU time dropped to 807.9 CPU seconds. This is roughly half the CPU time required by Esposito and Floudas (1998) to solve the problem on an HP 9000 C160 machine (which, based on the SPECfp95 benchmark, is a slightly faster machine than the Sun Ultra 2/1300 used here). Even if the problem had been solved using the inverse-midpoint preconditioner after 2 CPU days, the improvement factor due to the use of the new methodologies described here is over two orders of magnitude. 


\subsection{Problem 4: Parameter Estimation in Reactor Modeling}

This is another problem from Kim et al. (1990) and Esposito and Floudas (1998). It is a parameter estimation problem using the EIV approach to estimate kinetic parameters for an irreversible, first-order reaction $\mathrm{A} \rightarrow \mathrm{B}$, using data from an adiabatic CSTR. There are ten data points for five measured state variables, namely the CSTR inlet and outlet temperatures, the outlet concentrations of A and B, and the inlet concentration of A. Complete details of the problem are given by Gau and Stadtherr (2000b), who formulate it as an unconstrained global optimization problem with 22 variables. Again a maximum-likelihood-based estimator is optimized by using interval-Newton to determine the stationary points in the optimization problem, and the effects of using both the new hybrid preconditioning strategy and the new real point selection scheme are considered.

This global optimization problem was successfully solved, with the results for computational performance shown in Table 5. As in the previous problem, when the inverse-midpoint preconditioner alone was used, the program was still executing after two CPU days and so was terminated without reaching a solution. However, when the hybrid preconditioning strategy was used the problem was solved in only 255.2 CPU seconds, and with the addition of the real point selection scheme, in only $28.8 \mathrm{CPU}$ seconds. Relative to the $2 \mathrm{CPU}$ days spent without reaching a solution when the inverse midpoint preconditioner was used, the improvement factor when using the new methodologies approaches four orders of magnitude. Again, the solution time (28.8 CPU seconds) compares very favorably with that reported by Esposito and Floudas (1998), who tried three different problem formulations, with a fastest solution time of 282.2 CPU seconds on an HP 9000 C160, which, as noted above, is a slightly faster machine than the Sun Ultra 2/1300.

\section{Concluding Remarks}

We have described here new methodologies for improving the efficiency of the interval-Newton approach for the reliable solution of difficult global optimization and nonlinear equation solving problems. In particular, we have presented a new hybrid preconditioning strategy, in which a simple pivoting preconditioner is used in combination with the standard inverse-midpoint method, and a new scheme for selecting the real point in formulating the interval-Newton equation. These techniques can be implemented with relatively little computational overhead, and lead to a large reduction in the number of subintervals that must be tested during the interval-Newton procedure. Tests on a variety of problems arising in chemical process 
modeling have shown that the new methodologies lead to substantial reductions in CPU time requirements, in many cases by multiple orders of magnitude. For each of the two parameter estimation examples, the interval-Newton solution of the global optimization problem was essentially intractable using the standard methodology; however, when the new strategies were applied, these problem could be solved easily.

The use of interval-Newton/generalized-bisection methods represents a powerful and general-purpose approach to the solution of a number of difficult global optimization and nonlinear equation solving problems, such as arise in chemical process engineering. Continuing improvements in methodology, together with advances in software (e.g., compilers that treat intervals as a standard data type) and hardware (e.g., faster processors and parallel computing) will make this an increasingly attractive problem solving tool.

\section{Acknowledgments}

This work has been supported in part by the donors of The Petroleum Research Fund, administered by the ACS, under Grant 35979-AC9, by the National Science Foundation Grant EEC97-00537-CRCD, and by the Environmental Protection Agency Grant R826-734-01-0. 


\section{References}

Adjiman C. S., Dallwig, S., Floudas, C. A. \& Neumaier, A. (1998a) A global optimization method, alpha BB, for general twice-differentiable constrained NLPs - I. Theoretical advances. Comput. Chem. Eng., 22, 1137.

Adjiman C. S., Androulakis, I. P. \& Floudas, C. A. (1998b) A global optimization method, alpha BB, for general twice-differentiable constrained NLPs - II. Implementation and computational results. Comput. Chem. Eng., 22, 1159.

Alefeld, G. E., Potra, F. A., \& Volker, W. (1998) Modifications of the interval-Newton-method with improved asymptotic efficiency. BIT, 38, 619 .

Balaji, G. V. \& Seader, J. D. (1995) Application of interval-Newton method to chemical engineering problems. AIChE Symp. Ser., 91(304), 364.

Chen, H. S. \& Stadtherr, M. A. (1981). A modification of Powell's dogleg method for solving systems of nonlinear equations. Comput. Chem. Eng., 5, 143.

Dinkel, J. J., Tretter, M., \& Wong D. (1991). Some implementation issues associated with multidimensional interval Newton methods. Computing, 47, 29.

Esposito, W. R. \& Floudas, C. A. (1998). Global optimization in parameter estimation of nonlinear algebraic models via the error-in-variables approach. Ind. Eng. Chem. Res., 37, 1841.

Gau, C.-Y., Brennecke, J. F. \& Stadtherr, M. A. (2000a) Reliable parameter estimation in VLE modeling. Fluid Phase Equilib., 168, 1.

Gau, C.-Y. \& Stadtherr, M. A. (2000b) Reliable nonlinear parameter estimation using interval analysis: Error-in-variable approach. Comput. Chem. Eng., 24, 631.

Gan, Q., Yang, Q., \& Hu, C. -Y. (1994) Parallel all-row preconditioned interval linear solver for nonlinear equations on multiprocessors. Paral. Comput., 20, 1249.

Granvilliers, L. \& Hains, G. (2000). A conservative scheme for parallel interval narrowing. Inform. Process. Lett., 74, 141. 
Hansen, E. (1965) Interval arithmetic in matrix computations, part I. J. SIAM Numer. Anal., 2, 308.

Hansen, E. R. (1992). Global Optimization Using Interval Analysis, Marcel Dekkar, New York, NY.

Hansen, E. R. (1997) Preconditioning linearized equations. Computing, 58, 187.

Hansen, E. R. \& Greenberg, R. I. (1983). An interval Newton method. Appl. Math. Comput., 12, 89.

Hansen, E. R. \& Sengupta S. (1981) Bounding solutions of systems of equations using interval analysis. BIT, 21, 203.

Harding, S. T., Maranas, C. D., McDonald, C. M. \& Floudas, C. A. (1997) Locating all homogeneous azeotropes in multicomponent mixtures. Ind. Eng. Chem. Res., 36, 160.

Harding, S. T. \& Floudas, C. A. (2000) Locating all heterogeneous and reactive azeotropes in multicomponent mixtures. Ind. Eng. Chem. Res., 39, 1576.

Herbort, S. \& Ratz, D. (1997) Improving the efficiency of a nonlinear-system-solver using a componentwise Newton method. Technical Report 2/1997, Institut für Angewandte Mathematik, Universität Karlsruhe, Karsruhe, Germany.

Hu, C. -Y. (1990) Optimal Preconditioners for Interval Newton Methods, Ph.D. Thesis, University of Southwestern Louisiana, Lafayette, Louisiana.

Hua, J. Z., Brennecke, J. F \& Stadtherr, M. A. (1996) Reliable prediction of phase stability using an interval-Newton method. Fluid Phase Equilib., 116, 52.

Hua, J. Z., Brennecke, J. F. \& Stadtherr, M. A. (1998) Enhanced interval analysis for phase stability: cubic equation of state models. Ind. Eng. Chem. Res., 37, 1519.

Jalali-Farahani, F. \& Seader, J. D. (2000) Use of homotopy-continuation method in stability analysis of multiphase, reacting systems. Comput. Chem Eng., 241997.

Jansson, C. (2000) Convex-Concave extensions. BIT, 40, 291.

Kearfott, R. B. (1990) Preconditioners for the interval Gauss-Seidel method. SIAM J. Numer. Anal., 27, 804. 
Kearfott, R. B. (1991) Decomposition of arithmetic expressions to improve the behavior of interval iteration for nonlinear systems. Computing, 47, 169.

Kearfott, R. B. (1996). Rigorous Global Search: Continuous Problems, Kluwer Academic Publishers, Dordrecht, The Netherlands.

Kearfott, R. B. (1997) Empirical evaluation of innovations in interval branch and bound algorithms for nonlinear systems. SIAM J. Sci. Comput., 18, 574.

Kearfott, R. B. \& Novoa, M. (1990). INTBIS, a portable interval Newton/bisection package. ACM Trans. Math. Softw., 16, 152.

Kearfott, R. B., Hu, C.-Y., \& Novoa, M. (1991). A review of preconditioners for the interval Gauss-Seidel method. Interval Computing, 1, 59.

Kearfott, R. B., Dawande, M., Du, K.-S. \& Hu. C.-Y. (1994) INTLIB, a portable FORTRAN 77 interval standard function library. ACM Trans. Math. Softw., 20, 447.

Kearfott, R. B., Dian, J. \& and Neumaier, A. (2000) Existence verification for singular zeros of nonlinear systems. SIAM J. Numer. Anal., 38, 360.

Kim, I., Liebman, M., \& Edgar, T. (1990). Robust error-in-variables estimation using nonlinear programming techniques. AIChE J., 36, 985.

Krawczyk, R. (1969) Newton-Algorithmen zur Bestimmumg von Nullstellen mit Fehlershranken. Computing, 4, 187.

Kuno, M. \& Seader, J. D. (1988) Computing all real solutions to systems of nonlinear equations with a global fixed-point homotopy. Ind. Eng. Chem. Res., 27, 1320.

Lucia, A. \& Liu, D. L. (1998) An acceleration method for dogleg methods in simple singular regions. Ind. Eng. Chem. Res., 37, 1358.

Madan, V. P. (1990) Interval Newton method: Hansen-Greenberg approach - Some procedural improvements. Appl. Math. Comput., 35263.

Maier, R. W., Brennecke, J. F. \& Stadtherr, M. A. (1998) Reliable computation of homogeneous azeotropes. AIChE J., 44, 1745. 
Maier, R. W., Brennecke, J. F. \& Stadtherr, M. A. (1998) Reliable computation of reactive azeotropes. Comput. Chem. Eng., 24, 1851.

Makino, K. \& Berz, M. (1999) Efficient control of the dependency problem based on Taylor model methods. Reliable

Computing, 5, 3.

Maranas, C. D. \& Floudas, C. A. (1995) Finding all solutions of nonlinearly constrained systems of equations. J. Global Optim., 7, 143.

McKinnon, K. I. M., Millar, C. G. \& Mongeau M. (1996) Global optimization for the chemical and phase equilibrium problem using interval analysis. In State of the Art in Global Optimization: Computational Methods and Applications, eds. C. A. Floudas \& P. M. Pardalos, Kluwer Academic Publishers, Dordrecht, The Netherlands.

Neumaier, A. (1990). Interval Methods for Systems of Equations, Cambridge University Press, Cambridge, UK.

Powell, M. J. D. (1970) A hybrid method for nonlinear equations. In Numerical Methods for Nonlinear Algebraic Equations, ed. P. Rabinowitz, Gordon and Breach, London, UK.

Ratschek, H. \& Rokne, J. (1984) Computer Methods for the Range of Functions. Halsted Press, New York, NY.

Ratz, D. (1994) Box-splitting strategies for the interval Gauss-Seidel step in a global optimization method. Computing, 53337.

Schnepper, C. A. \& Stadtherr, M. A. (1996) Robust process simulation using interval methods. Comput. Chem. Eng., 20, 187.

Shacham, M. \& Kehat, E. (1973) Converging interval methods for the iterative solution of a non-linear equation. Chem. Eng. Sci., 28, 2187.

Sørensen, J. M. \& Arlt, W. (1979-1987) Liquid-Liquid Equilibrium Data Collection, Volume V of Chemistry Data Series. DECHEMA, Frankfurt/Main, Germany. 
Stadtherr, M. A., Schnepper, C. A. \& Brennecke, J. F. (1995) Robust phase stability analysis using interval methods. AIChE Symp. Ser., 91(304), 356.

Stradi, B. A. (1999) Measurement and Modeling of the Phase Behavior of High Pressure Reaction Mixtures and the Computation of Mixture Critical Points, Ph.D. Thesis, University of Notre Dame, Notre Dame, IN.

Stradi, B. A., Brennecke, J. F., Kohn, J. P. \& Stadtherr, M. A. (2001) Reliable computation of mixture critical points. AIChE J, 47, 212.

Sun, A. C. \& Seider, W. D. (1995) Homotopy-continuation method for stability analysis in the global minimization of the Gibbs free energy. Fluid Phase Equilib., 103, 213.

Tessier, S. R., Brennecke, J. F. \& Stadtherr, M. A. (2000) Reliable phase stability analysis for excess Gibbs energy models. Chem. Eng. Sci., 55, 1785.

van Hentenryck, P., McAllester, D., \& Kapur, D. (1997) Solving polynomial systems using a branch and prune approach. SIAM J. Numer. Anal., 34, 797.

Wayburn, T. L. \& Seader, J. D. (1987) Homotopy-continuation methods for computer-aided process design. Comput. Chem. Eng., 11, 7.

Xu, G., Scurto, A. M., Castier, M., Brennecke, J. F. \& Stadtherr, M. A. (2000) Reliable computation of high pressure solid-fluid equilibrium. Ind. Eng. Chem. Res., 39, 1624.

Yamamura, K., Kawata, H. \& Tokue, A. (1998). Interval solution of nonlinear equations using linear programming. BIT, 38, 186.

Yamamura, K., \& Nishizawa, M. (1999). Finding all solutions of a class of nonlinear equations using an improved LP test. Japan J. Appl. Math., 16, 349. 
Table 1. Computational performance on Problem 1: Phase stability analysis for mixtures of acetic acid(1), benzene(2), furfural(3), and cyclohexane(4)

\begin{tabular}{|c|ccc|ccc|}
\hline & \multicolumn{2}{|c|}{ Number of Subintervals Tested } & \multicolumn{3}{c|}{ CPU Time $(\mathrm{s})^{*}$} \\
\cline { 2 - 7 } Feed $\left(z_{1}, z_{2}, z_{3}, z_{4}\right)$ & IMP & HP & ImpFac & IMP & HP & ImpFac \\
\hline$(0.25,0.25,0.25,0.25)$ & 4,209 & 906 & 4.6 & 1.29 & 0.37 & 3.5 \\
$(0.05,0.20,0.35,0.40)$ & 7,095 & 849 & 8.4 & 2.19 & 0.36 & 6.1 \\
$(0.05,0.21,0.34,0.40)$ & 8,491 & 919 & 9.2 & 2.61 & 0.38 & 6.9 \\
$(0.05,0.22,0.33,0.40)$ & 10,955 & 1,016 & 10.8 & 3.41 & 0.42 & 8.1 \\
$(0.05,0.23,0.32,0.40)$ & 26,947 & 1,247 & 21.6 & 8.27 & 0.52 & 15.9 \\
\hline
\end{tabular}

${ }^{*}$ CPU time on Sun Ultra 2/1300 workstation 
Table 2. Computational performance on Problem 1: Phase stability analysis for mixtures of acetic acid(1), benzene(2), furfural(3), cyclohexane(4), and water(5)

\begin{tabular}{|c|ccc|ccc|}
\hline \multirow{2}{*}{ Feed $\left(z_{1}, z_{2}, z_{3}, z_{4}, z_{5}\right)$} & \multicolumn{2}{|c|}{ Number of Subintervals Tested } & \multicolumn{3}{c|}{ CPU Time (s) } \\
\cline { 2 - 7 } & IMP & HP & ImpFac & IMP & HP & ImpFac \\
\hline$(0.20,0.20,0.20,0.20,0.20)$ & 311,745 & 18,194 & 17.1 & 143.68 & 12.21 & 11.7 \\
$(0.20,0.25,0.20,0.15,0.20)$ & 352,054 & 19,447 & 18.1 & 161.25 & 13.06 & 12.3 \\
$(0.20,0.25,0.25,0.15,0.15)$ & 647,875 & 23,678 & 27.4 & 296.40 & 16.25 & 18.2 \\
$(0.10,0.25,0.25,0.15,0.25)$ & 114,753 & 14,537 & 7.9 & 55.28 & 9.59 & 5.8 \\
$(0.15,0.25,0.25,0.10,0.25)$ & 214,395 & 17,280 & 12.4 & 100.63 & 11.55 & 8.7 \\
\hline
\end{tabular}

${ }^{*}$ CPU time on Sun Ultra 2/1300 workstation 
Table 3. Computational performance on Problem 1: Phase stability analysis for mixtures of benzene(1), cyclohexane(2), 1,2-ethanediol(3), furfural(4), heptane(5), and water(6).

\begin{tabular}{|c|ccc|ccc|}
\hline \multirow{2}{*}{ Feed $\left(z_{1}, z_{2}, z_{3}, z_{4}, z_{5}, z_{6}\right)$} & \multicolumn{2}{|c|}{ Number of Subintervals Tested } & \multicolumn{3}{c|}{ CPU Time (s) } \\
\cline { 2 - 7 } & IMP & HP & ImpFac & IMP & HP & ImpFac \\
\hline$(0.10,0.25,0.10,0.25,0.10,0.20)$ & $69,567,565$ & 153,876 & 452.1 & 50217.3 & 152.0 & 330.4 \\
$(0.25,0.10,0.10,0.10,0.25,0.20)$ & $9,748,253$ & 122,781 & 79.4 & 7077.5 & 119.9 & 59.0 \\
$(0.10,0.20,0.10,0.25,0.25,0.10)$ & $205,932,188$ & 148,331 & 1388 & 146903 & 147.7 & 994.6 \\
$(0.20,0.10,0.10,0.30,0.10,0.20)$ & $81,081,374$ & 176,206 & 460.2 & 57900.7 & 176.1 & 328.8 \\
$(0.10,0.10,0.10,0.20,0.25,0.25)$ & $68,597,673$ & 118,569 & 578.5 & 48986.6 & 116.4 & 420.8 \\
\hline
\end{tabular}

${ }^{*}$ CPU time on Sun Ultra 2/1300 workstation 
Table 4. Computational performance on Problem 2: Computation of mixture critical points.

\begin{tabular}{|c|ccc|ccc|}
\hline \multirow{2}{*}{ Mixture } & \multicolumn{3}{|c|}{ Number of Subintervals Tested } & \multicolumn{3}{c|}{ CPU Time (s) } \\
\cline { 2 - 7 } & IMP & HP (ImpFac) & HP/RP (ImpFac) & IMP & HP (ImpFac) & HP/RP (ImpFac) \\
\hline 1 & 405,623 & $72,157(5.6)$ & $32,296(12.6)$ & 154.9 & $30.8(5.0)$ & $20.7(7.5)$ \\
2 & $4,068,420$ & $1,084,110(3.8)$ & $483,227(8.4)$ & 2094.1 & $658.8(3.2)$ & $406.5(5.2)$ \\
3 & $7,351,170$ & $3,416,481(2.2)$ & $2,121,333(3.4)$ & 4909.1 & $2539.2(1.9)$ & $1796.2(2.7)$ \\
\hline
\end{tabular}

${ }^{*}$ CPU time on Sun Ultra 2/1300 workstation 
Table 5. Computational performance on Problems 3 and 4: Parameter estimation using EIV approach.

\begin{tabular}{|c|ccc|}
\hline \multirow{2}{*}{ Problem } & \multicolumn{3}{|c|}{ CPU Time (s) ${ }^{*}$} \\
\cline { 2 - 4 } & IMP & HP (ImpFac $\ddagger)$ & HP/RP (ImpFac $\ddagger)$ \\
\hline \multirow{2}{*}{3} & $>172,800 \dagger$ & $1504.2(>115)$ & $807.9(>214)$ \\
4 & $>172,800 \dagger$ & $255.2(>677)$ & $28.8(>6000)$ \\
\hline
\end{tabular}

${ }^{*}$ CPU time on Sun Ultra 2/1300 workstation $\dagger$ Program was terminated after 2 CPU days without reaching solution $\ddagger$ Relative to 2 CPU days spent without reaching solution 